\title{
Application of Wavelet Transformation for the Identification of High Frequency Spurious Signals in Step Down DC - DC Converter Circuits Experiencing Intermittent Chaotic Patterns
}

\author{
D. Giaouris, B. Zahawi, G. El-Murr and V. Pickert \\ School of EECE, University of Newcastle upon Tyne, Newcastle upon Tyne NE1 7RU, UK \\ damian.giaouris@ncl.ac.uk
}

Keywords: Wavelet transformation, dc-dc converters, intermittent chaos

\begin{abstract}
Intermittent chaotic phenomena can appear in a dc-dc step down converter when the later is coupled with spurious high frequency signals. It is therefore important to understand why and how these phenomena occur and what is their relationship with the frequency characterises of the spurious injected signals. This paper uses a fast wavelet transform to isolate the frequency of these signals even when the converter is in a chaotic or subharmonic mode of operation.
\end{abstract}

\section{Introduction}

The stability and bifurcation properties of a buck converter (Fig. 1) have been extensively studied in many publications and are still an active and challenging research topic. It is well known that by varying the system's parameters it is possible to cause slow time scale instabilities to the system which can be studied through its average model. It is also known that fast scale instabilities may also occur which can not be determined by the previous method of analysis [1 - 2]. These instabilities are generally referred to as subharmonic and chaotic patterns. These phenomena have been extensively studied and analysed, however, the usual engineering practice is to try to avoid them by suitably adjusting the system's parameters [3]. Furthermore, it has recently been reported that the stable behaviour of the system can be prejudiced if the converter is influenced by external factors, usually described as an unwanted coupling of the system with spurious signals. Assuming these signals to be purely sinusoidal, Tse, Zhou, Lau, and Qiu have shown, through experimental tests and numerical simulations, that this spurious coupling can cause intermittent chaotic and subharmonic windows of operation [4].

The frequency of these spurious signals greatly influences the frequency and the duration of the intermittent chaotic and subharmonic phenomena. Knowledge of this frequency is therefore of great importance when designing and implementing suitable chaos control strategies [5]. In this paper a Fast Wavelet Transform (FWT) is used to identify this frequency. By using this analysis it is possible to isolate and calculate the time varying frequency component of the output voltage of the converter and hence the frequency of the spurious signals.

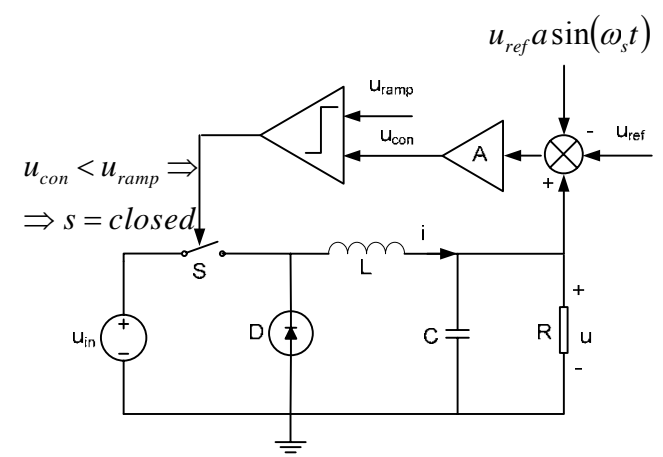

Fig. 1: Buck converter coupled with high frequency signal

\section{Wavelet Analyses}

Wavelet analysis is employed in a large area of applications in signal processing including multiresolution analysis used in computer vision, subband coding developed for speech and image compression, and recently fault detection in electric machines [6]. Wavelets have been a very popular topic in many scientific and engineering research papers. Some view wavelets as a new basis for representing functions, some consider it as a technique for time-frequency analysis, and others think of it as new mathematical subject. However from the signal analyst's point of view, wavelet analysis is a decomposition of the signal on a family of time-frequency analysing signals.

The history of wavelets begins with the Fourier Transform (FT), when in the early 1800 s the French mathematician Jean Fourier discovered that he could represent periodic functions in terms of sine and cosine waves. The Fourier transform of $x(t)$ is defined as:

$$
X(f)=\int_{-\infty}^{\infty} x(t) e^{-j 2 \pi f t} d t
$$

In (1) the inner product of the signal with the sine wave is computed over an infinite duration. Thus, the FT gives the frequency spectrum of a signal, but it gives no information about where in time those spectral components appear. As a result, the FT works well if the signal is stationary, i.e. when its spectrum is time invariant. In practice, the majority of signals are nonstationary. Therefore, an analysis adapted to nonstationary signals requires more than the Fourier transform. Short Time Fourier Transform (STFT) was introduced to analyse signals in time-frequency domain. 
STFT is an extension of the Fourier transform, where the signal is divided into time sections, as shown in Fig. 3. The signal within each section is considered stationary and the FT applied in each section. A windowed function $\mathrm{w}(t-b)$ is used for time-localization, where $b$ is used to translate the window over the whole signal. The STFT of $x(t)$ is represented as follows:

$$
X_{\text {STFT }}(b, f)=\int_{T}\left[x(t) w^{*}(t-b)\right] e^{-j e \pi f t} d t
$$

The width of the window must be equal to the interval of the signal where it is considered stationary. Once a window has been chosen for the STFT, the time-frequency resolution is fixed over the entire time-frequency plane (Fig. 2a). However if the signal is composed of short time high frequency signals associated with long stationary signal components, then each type of component can be analysed with good time resolution or frequency resolution, but not both. For example, if the window has an infinite length, then it is similar to Fourier transform, which gives a perfect frequency resolution, but no time information. Furthermore, to obtain a stationary signal the window's width must be narrow, the narrower the window the better is the time resolution but poorer is the frequency resolution. The choice of an appropriate window function is therefore a problem when using the STFT. Wavelet Transform (WT) is a technique introduced to solve the resolution problem by varying the windows' width, giving good time resolution and poor frequency resolution by contracting the window's width, and good frequency resolution and poor time resolution by dilating the window. The continuous wavelet transform of $x(t)$ is defined as:

$$
X_{W}(a, b)=\int_{-\infty}^{\infty} \Psi_{a, b}^{*}(t) x(t) d t
$$

This equation shows how a function $x(t)$ is decomposed into a set of basis functions $\psi_{a, b}^{*}(t)$, called the wavelets.

$$
\Psi_{a, b}(t)=\frac{1}{\sqrt{a}} \Psi\left(\frac{t-b}{a}\right)
$$

where, $a$ is the scale factor and $b$ is the translation factor.

Theoretically the continuous WT is infinitely redundant, thus the calculated transform contains large amount of unnecessary information. What's more, $a$ and $b$ are varying continuously therefore an infinite number of wavelet are inserted in the analysis of the signal. In practice, it is more convenient to remove unnecessary information and reduce the number of wavelets without loosing the essential information. To achieve this, the discrete wavelet transform (DWT) has been suggested (Fig. 2b). In a discrete wavelet transform, a wavelet is translated and dilated by discrete values, thus the continuous translation and dilation factors become discrete $a=a_{0}^{m}, b=n b_{0}$ and equation (4) is rewritten as:
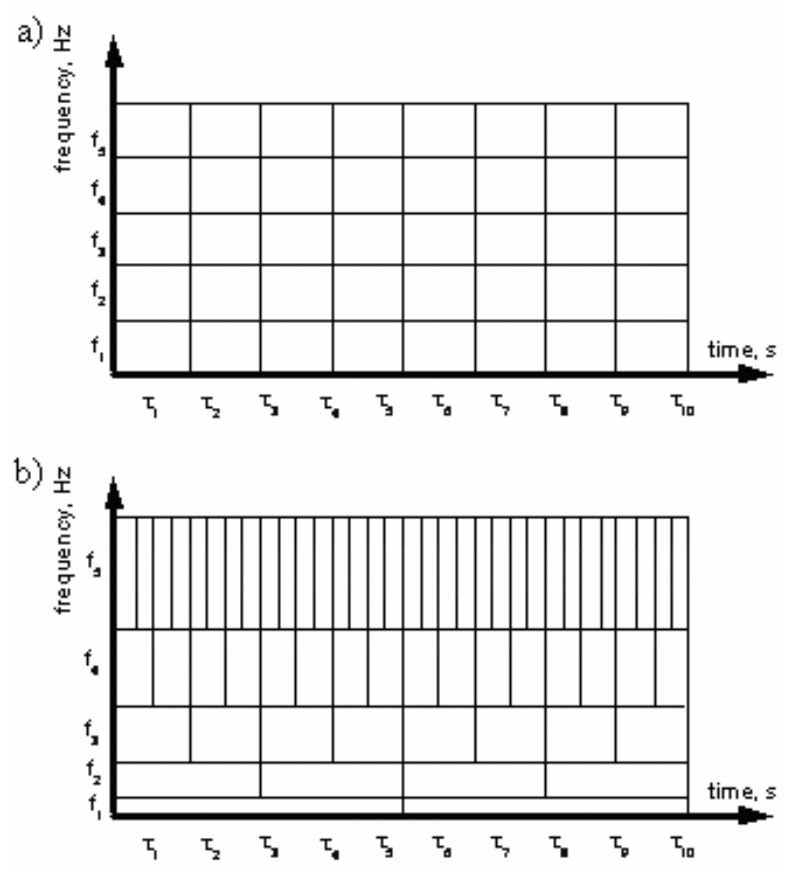

Fig. 2: Time-frequency plane a) STFT, b) DWT

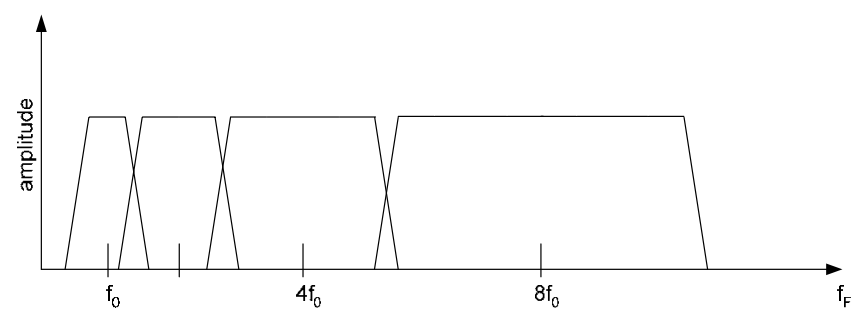

Fig. 3: Logarithmic coverage of the freq. spectrum of the WT

$$
\Psi_{m, n}(t)=\frac{1}{\sqrt{a_{0}^{m}}} \Psi\left(\frac{t}{a_{0}^{m}}-n b_{0}\right)
$$

where $m, n$ are positive integers, $a_{0}>1$, and $b_{0} \neq 0$.

By choosing $a_{0}=2$ and $b_{0}=1$ it is possible to construct orthonormal functions [7] from the prototype wavelet function $\Psi_{m, n}(t)$, which can be used to analyse a signal (Fig.

3) Those basis functions are called, the mother wavelet $\psi_{m, n}(t)$ and the scaling function $\varphi_{m, n}(t)$.

$$
\begin{aligned}
& \psi_{m, n}(t)=\frac{1}{\sqrt{2^{m}}} \psi\left(\frac{t}{2^{m}}-n\right) \\
& \varphi_{m, n}(t)=\frac{1}{\sqrt{2^{m}}} \varphi\left(\frac{t}{2^{m}}-n\right)
\end{aligned}
$$

Consequently a signal $x(t)$ can be decomposed into linear combinations of scaling functions and wavelets : 


$$
x(t)=\sum_{n=-\infty}^{\infty} c_{m, n} \varphi_{m, n}(t)+\sum_{m=0}^{\infty} \sum_{n=-\infty}^{\infty} d_{m, n} \psi_{m, n}(t)
$$

where $c_{m, n}$ and $d_{m, n}$ are the approximation and detailed coefficients, respectively.

\section{Detection of Spurious Signals}

The Fast Wavelet Transform (FWT) or Multiresolution Analysis gives a fast and simple method of decomposing the signal into various scales, by considering the scaling function and the mother wavelet as a lowpass and highpass FIR filters, respectively. Thus the FWT is simply convoluting the signal with those filters to obtain the empirical wavelet coefficients. The coefficients that result from the convolution of the signal with the associated low pass filter are called approximations and the coefficients that result from the high pass filtering are called details. The Fast Wavelet Transform representation provides a hierarchical framework for interpreting the signal, as demonstrated in Fig. 4.

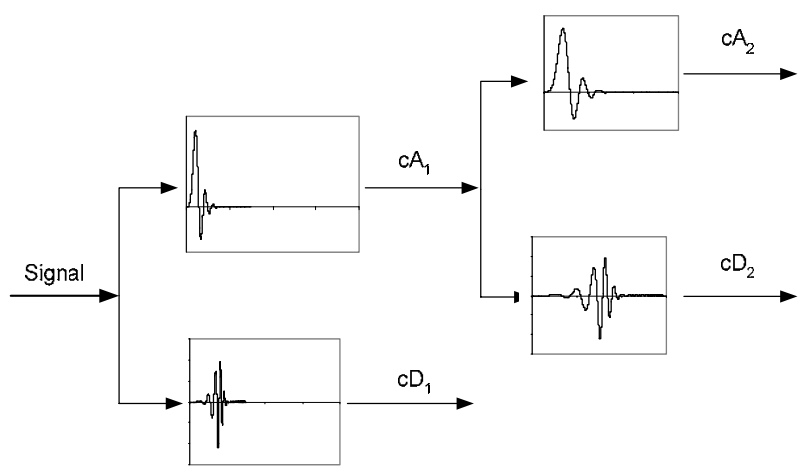

Fig. 4: Fast Wavelet Transform.

The resulting coefficients can be processed to compress, denoise or even isolate specific frequency components. The common method for signal denoising based on wavelets, as proposed by Donoho [8], can be broken up into 3 separate steps:

1) Use the hierarchical filter decomposition.

2) Assume that the noise components will have small amplitudes compared to the useful signals. Hence, a threshold technique may be applied to eliminate unwanted small coefficients.

3) Reconstruct the signal by inverting the hierarchical filters.

In Fig. 5 the behaviour of the buck converter is shown for various frequencies of the spurious signal, which is assumed to be purely sinusoidal [4]. A slight modification of the previous denoising technique is applied to the output voltage of the converter by using a common Daubechies 7 "db7"wavelet. The wavelet analysis at different scales of decomposition isolated a low frequency sine wave whose frequency equals the difference between the frequency of the spurious noise signal and that of the converter ramp signal.
At the reconstruction stage, all coefficients that had different frequency information were ignored and hence the output of the wavelet scheme included only one signal which looks very closely to the previous low frequency sine wave, as demonstrated in Fig. 6, which clearly shows that this signal is always present in the output voltage and that it is simply slightly distorted when the intermittent window occurs.

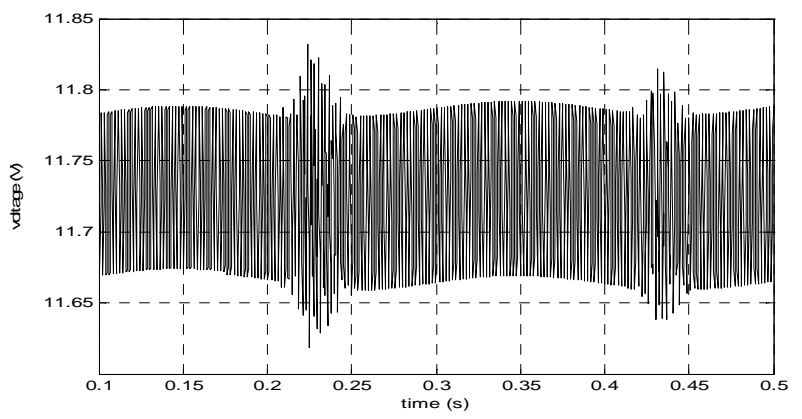

Fig. 5a: Converter output voltage, spurious signal frequency at $2505 \mathrm{~Hz}$

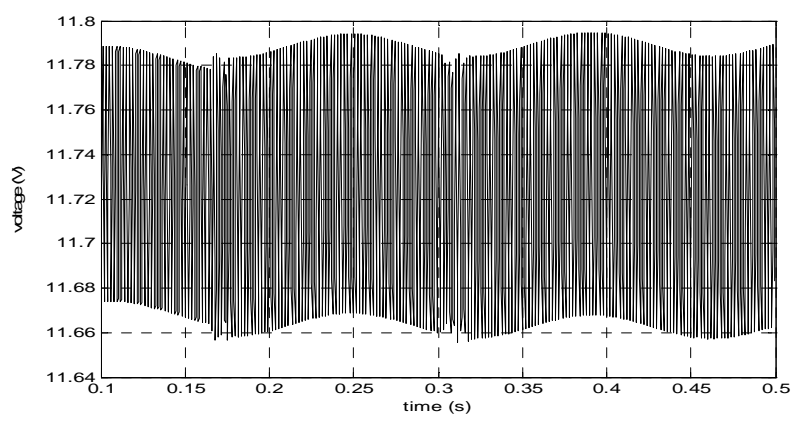

Fig. 5b: Converter output voltage, spurious signal frequency at $2507 \mathrm{~Hz}$

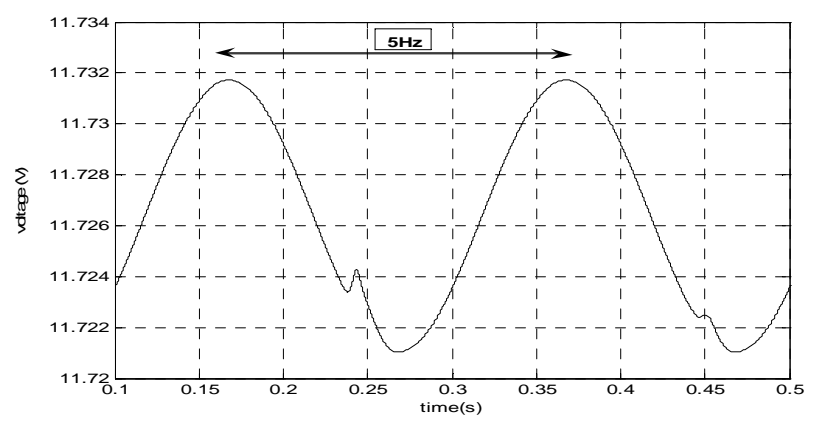

Fig. 6a: Wavelet analysis output, spurious signal at $2505 \mathrm{~Hz}$

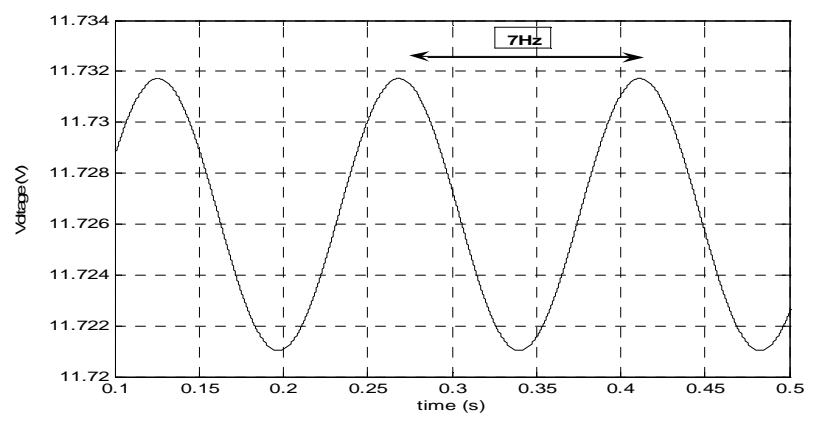

Fig. 6b: Wavelet analysis output, spurious signal at $2507 \mathrm{~Hz}$ 
To determine numerically the value of the intermittent frequency, the intermittent signal is passed through positive and negative comparators to measure the time difference $\Delta t$ between two successive peaks of the signal, allowing the period and hence the frequency of the signal to be calculated (Fig. 7).

(a)

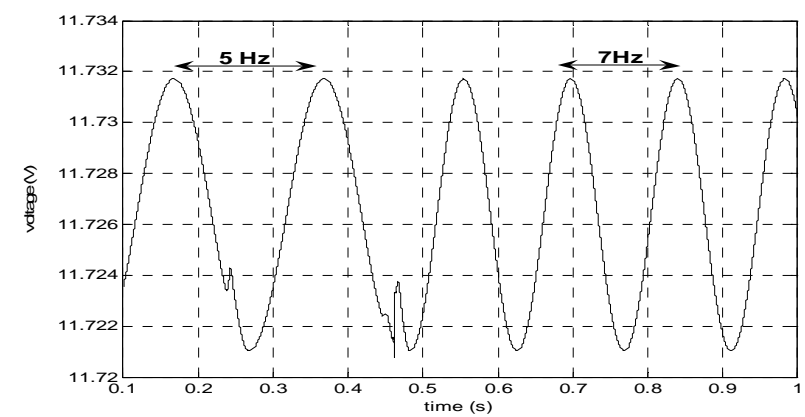

(b)

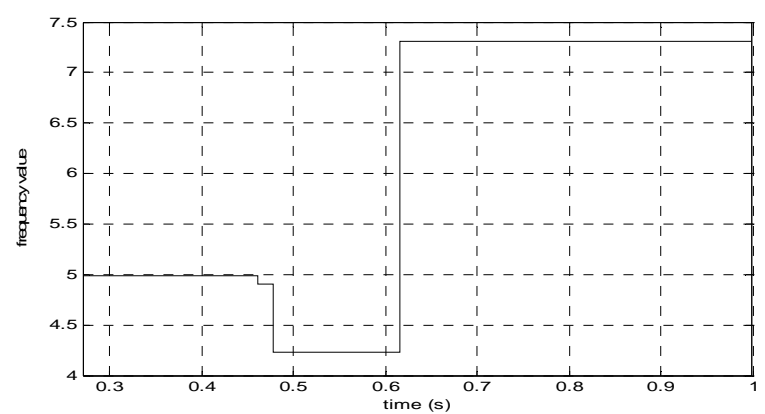

Fig. 7: Frequency identification of the spurious signal when its frequency s suddenly changed from $2505 \mathrm{~Hz}$ to $2507 \mathrm{~Hz}$, a) actual signal, b) identified frequency.

\section{Conclusions}

The Fast Wavelet Transform (FWT), based on successive approximations of the signal at different scales, has been used to identify the frequency of spurious noise signals that can drive dc-dc converter circuits into intermittent subharmonic or chaotic behaviour. By using this analysis, it is possible to produce a time frequency plane of the signal allowing the time varying frequency component of the output voltage of the converter and hence the frequency of the spurious signals to be isolate and calculate. The analysis provides a means of identifying the offending frequencies and hence provides a means of compensating for their effects.

\section{References}

[1] S. Banerjee, and G. C. Verghese, Nonlinear phenomena in power electronics, attractors, bifurcations, chaos and nonlinear control, IEEE Press, 2001, ISBN 0780353838

[2] D. Giaouris;B. Zahawi;V. Pickert. On the Derivations of the Monodromy Matrix of the Buck Converter. International Conference on Technology and Automation, ICTA05, Thessalonica, Greece, 2005

[3] D. C. Hamill, and D. J. Jefferies, "Subharmonics and chaos in a controlled switched-mode power converter", IEEE Transactions on Circuits and Systems, vol. 35, no. 8, pp. 1059 - 1061, August 1988

[4] C. K. Tse, Y. Zhou, F. C. M. Lau, and S. S. Qiu, "Intermittent chaos in switching power supplies due to unintended coupling of spurious signals," IEEE Power electronics specialists conference, 2003, Acapulco, Mexico, pp. 642-647

[5] Y. Zhou, C. K. Tse, S. S. Qiu, and F. C. M. Lau, "Applying resonant parametric perturbation to control chaos in the buck dc/dc converter with phase shift and frequency mismatch considerations", Int. Journal of Bifurcation and Chaos, vol. 13, no. 11, pp. 3459-3471, 2003

[6] H. Douglas, P. Pillay, and A. K. Ziarani "A New Algorithm for Transient Motor Current Signature Analysis Using Wavelet", IEEE Transactions on industry applications, vol.40, no.5, pp.1361-1368, 2004.

[7] I. Daubechies, "The wavelet transform, time-frequency localization and signal analysis", IEEE Transactions on Information Theory, vol. 36, no. 5, pp. 961 - 1005, 1990

[8] D. L. Donoho "De-Noising by Soft-Thresholding", IEEE Transactions on information theory, vol. 41, no.3, pp.613-627, 1995 